Revista del Centro de Investigación de la Universidad La Salle

Vol. 12, No. 48, julio-diciembre, 2017: 9-30

DOI: http://dx.doi.org/10.26457/recein.v12i48.1508

\title{
Reflexiones en torno a la relación entre pedagogía, currículo y pensamiento complejo
}

Reflections on the relationship between pedagogy, curriculum and complex

thinking

\author{
Emilio Roger Ciurana* \\ Universidad de Valladolid (España) \\ Cecilia Regalado Lobo \\ Universidad de Valladolid (España)
}

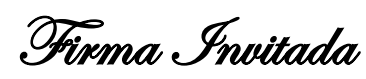

Fecha de recepción: 07 de septiembre de 2017

Fecha de aceptación: 09 de diciembre de 2017

Disponible en línea: 28 de febrero de 2018

\section{Resumen:}

Los temas que serán abordados en el siguiente artículo están relacionados con la necesidad de pensar de un modo inter y transdisciplinar, complejo, con la finalidad de alcanzar el impacto que el pensamiento necesita en relación con los problemas actuales que atañen a la enseñanza y la pedagogía. Una de las cuestiones medulares en torno a los problemas de la

\footnotetext{
*E-mail: emiliorogerciurana@outlook.com
} 
situación actual de la educación tiene que ver con la producción de currículums estrictos, preocupados esencialmente por la aplicación de principios esquemáticos para calificar el aprendizaje del sujeto antes que por experimentar progresivamente su inmersión en los procesos de aprendizaje reales y de impacto. Para acercarnos a esta dimensión de la pedagogía, donde existen mecanismos mixtos que deben ser interrelacionados una y otra vez superados, recuperados y puestos en nuevos ambientes, todos ellos dentro de un ambiente cambiante, se exige la adaptación a lo inesperado, a las sorpresas del aprendizaje, dentro de situaciones volátiles, líquidas y a partir de una ontología capaz de ser reticular y móvil.

Palabras clave: Currículo; Pensamiento complejo; Pedagogía; Creatividad; dia-logo. 


\section{Abstract}

The topics that will be addressed in the following paper, are related to the need to think in an inter transdisciplinary and complex way, with the purpose of reaching the impact that the thought needs in relation to the current problems that concern to the education and the pedagogy. One of the central issues surrounding the problems of the current situation of education has to do with the production of strict curricula, essentially concerned with the application of schematic principles to qualify the learning of students rather than experiencing progressively their immersion in the real learning processes and its impact. To approach this dimension of pedagogy, where there are mixed mechanisms that must be interrelated over and over again, overcome, recovered and placed in new environments; all of them within a changing environment, adaptation to the unexpected and the surprises of learning is required, within volatile and liquid situations, and from an ontology capable of being reticular and mobile.

Keywords: Curriculum; Complex thinking; Pedagogy; Creativity; dia-logue. 


\section{Introducción}

Los autores de este texto partimos de una idea que nos parece básica y previa a toda reflexión referida al quehacer curricular. Sobre todo, en un tiempo como el nuestro, en el que se habla tanto de innovación educativa, de planificación, de uso de las tecnologías aplicadas a la educación; nos parece necesario rescatar las que creemos son preguntas originales que afectan a la educación y contextualizarlas en nuestras circunstancias. Después vendrán las técnicas que puedan ayudar a poner en práctica las ideas. El diseño de algo debe responder a una intención, a una interrogación, a una necesidad, a una problemática.

\section{Una nueva visión de la educación: repensando la educación}

Comenzamos por anticipar que para nosotros la educación es, entre otras cosas, pero de modo fundamental, un laboratorio del pensamiento, esto es una puesta en práctica de una filosofía sobre cómo habilitar al ser humano para una adecuada inserción en su contexto (glocal), en la ciudad (polis). Es una práctica auto-eco-reorganizadora porque la experiencia, los resultados del proceso teórico/práctico, puede aportarnos información significativa si reflexionamos sobre la relación que establecemos con el contexto. Esta práctica reflexiva nos puede permitir observar posibles modificaciones en nuestra manera de pensar y actuar en el mundo para lograr una mayor pertinencia respecto a los problemas reales, no abstractos, no simulados. Dicho de otro modo, pensamos en una institución educativa no autorreferencial sino auto-eco-reorganizadora, insertada en una sociedad y tiempo concretos. Una institución educativa que no reproduzca abstracciones (como si viviese en un espacio controlado), sino que se engarce con la sociedad real y en la que los contenidos que se impartan estén articulados a las problemáticas reales, porque la persona que estudia tiene ante sí una sociedad y un mundo que le plantea desafíos y cuestiones a resolver y, en ese sentido, cuanto más pertinentes sean las soluciones a los retos del presente más calidad de vida es posible construir. Una institución educativa, en síntesis, sensible. Nos atrevemos a decir que toda educación que separe el pensar de la experiencia es un fiasco. Por otra parte, hablamos de educación como proyecto humano amplio que incluye a todos independientemente de las 
formaciones particulares de lo que son las licenciaturas o los grados. En todo caso lo que decimos se puede (a nivel epistemológico) aplicar a la formación particular de cualquier grado.

También es verdad que hoy en día muchas propuestas educativas con diseños técnicos ad hoc, tienen clara la intención de esa educación separada y fragmentada de la experiencia amplia del sujeto en el contexto: educar para el mercado, por ejemplo; disolver lo humanístico para potenciar solo lo que responde a lo circunstancial dentro de la esfera neoliberal y materialista en la que estamos insertados; despolitizar al ser humano, esto es, impedir que el ser humano sea un ciudadano en una comunidad política de iguales; insertarle una información abstracta y controlada desajustada respecto del contexto... Reducirlo a un buen técnico, un buen consumidor, un buen elector (dentro de menús diseñados sin contar con él), un buen trabajador que se adapta a los cambios tecnológicos sin comprender el por qué y para qué de los cambios, ya que no comprende el mundo en el que vive.

Todo ello dentro de un marco ideológico que privilegia la velocidad, la rapidez, y que potencia el innovar en tecnologías sin preocuparse por innovar ${ }^{1}$ en la estructura de pensamiento que posibilite la creatividad del sujeto en contextos que constantemente producen novedades (emergencias). De hecho, hoy en día cuando se usa la palabra "excelencia" aplicada a la educación la cosa trata más de la excelencia en adaptarnos a normas impuestas por especialistas y grupos de presión que al hecho de ser excelentes en el pensar. Lo excelente hoy responde a la capacidad de ejecutar y reproducir bien programas impuestos (muchas veces de modo externo al ámbito educativo) y, en ese sentido, alguien saldrá bien evaluado si ejecuta bien esos programas. Prácticamente la calidad y la excelencia en la educación tienen como baremo de medida la utilidad para los intereses del mercado tecno-industrial y laboral. (intereses que pueden ser bastante cambiantes, y que hacen que la instrucción recibida, en un momento dado, en relación a las necesidades del mercado ${ }^{2}$ se haga muy pronto inadecuada debido a la rapidez de los cambios y la volatilidad de los mercados). Para ello se proporcionan plantillas e indicadores elaborados por expertos y a partir de estas plantillas e indicadores se marcan fortalezas, debilidades a corregir... en torno a marcos de intereses... pareciera que excelencia y control son inseparables. 
Las preguntas originales que se plantearon los filósofos que comenzaron a reflexionar sobre la educación (la preocupación filosófica, pedagógica y política surgen juntas en la Antigüedad) eran preguntas que trataban de resolver, por ejemplo, el dilema ser/tener. ¿Educar para ser? ¿Educar para tener? Ser qué. Tener qué. Ello implicaba también reflexionar (lo mismo nos concierne hoy, porque se trata, como siempre, se aprende a ser) sobre qué tipo de sociedad deseamos, dónde estamos en este momento y qué racionalidad social e individual recubre $\mathrm{u}$ orienta nuestras acciones. ¿Una racionalidad tecnológica, descontextualizada y formalista? ¿Una racionalidad basada en el modelo del "actor racional"? Sin duda es más fácil programar, adiestrar y/o amaestrar por medio de la educación a individuos irreflexivos que ayudarles (proporcionarles estrategias) a ejercer la habilidad para la creación y el reaprendizaje en un contexto/tiempo que no se condensa, sino que fluye; un contexto/tiempo incierto por caótico y que constantemente necesita de la intervención reflexiva del sujeto.

Una de las conclusiones que pretendemos aportar es la siguiente: toda acción racional es acción metodológica y no una acción programática o programada. ¿Por qué metodológica? Porque es reflexiva. Y es reflexiva porque toda teoría, todo pensar se revisa en el transcurso de la acción y toda acción necesita de un pensar y un teorizar. Método y materia sobre la que trabaja están en una constante retroacción. Se trata de un proceso dinámico co-productor. En síntesis, se trata de habilitar al sujeto contra la rutinización de la costumbre, si queremos usar la palabra "rutinización" en el sentido que le daba Dewey ${ }^{3}$ en sus reflexiones sobre la educación. Volveremos sobre ello, pero es bueno que siempre tengamos en cuenta que los hábitos y las rutinas pueden ayudar y facilitar las cosas en algún momento, pero también pueden esclavizar ${ }^{4}$. Los hábitos y las rutinas son de escasa ayuda cuando el medio es cambiante, móvil, líquido; esto es, cuando emerge lo complejo. Dicho de otro modo: una conducta programada ayuda si el medio es constante, pero puede ser muy perjudicial en contextos cambiantes o en sociedades pluriculturales. Donde emerge constantemente diversidad hay que tener estrategias de comprensión dialógica ${ }^{5}$. Por ello mismo la conexión entre filosofía y educación tiene como labor fundamental proporcionar respuestas adecuadas 
(partiendo de problemas bien planteados a partir de una buena hermenéutica del contexto) a las perturbaciones (inestabilidades, fluctuaciones) del medio. Idear posibilidades que se puedan materializar en una práctica. Posibilitar la gestión de lo anormal porque lo normal se gestiona de modo mecánico, rutinario.

Usamos la expresión de Bauman, se trata de aprender a caminar sobre arenas movedizas. Desde luego es difícil caminar sobre algo no sólido si tenemos poco sentido del equilibrio y poca capacidad estratégica a la hora de responder a los imprevistos movimientos bajo nuestros pies. Se nos ha educado para vivir en lo sólido sin enseñarnos que lo sólido es o son condensaciones temporales de materiales magmáticos. El problema fundamental entonces es un problema epistemológico. Como decía Bachelard (1981), todo discurso del método es un discurso de circunstancias (p.121). Se trata de aprender a gestionar el caos. Los programas solo gestionan la norma, nosotros debemos educarnos para gestionar lo no programado, lo indeterminado, con la conciencia de que no existen formas a priori para eliminar la incertidumbre $y$ por ello debemos fomentar nuestra capacidad estratégica/creativa6 ${ }^{6}$ Estrategia creativa, porque como bien dice E. Morin (2014): "la incertidumbre no se elimina, se negocia con ella" (p. 37), nunca podemos estar seguros de haber eliminado toda la incertidumbre porque no tenemos, salvo en casos triviales, un control total del medio.

Necesitamos, sigue diciendo Morin: "aprender a navegar en un océano de incertidumbres a través de archipiélagos de certezas" (p. 36). Ello implica que aprendamos a aprender de otro modo, que pensemos en otro tipo de ontología, que cambiemos de una ontología estática a una ontología de la relación y el devenir. Implica que pensemos en un mundo en el que las cosas están en un constante hacerse, reorganizarse. El mundo no está dado desde siempre, lo hacemos, rehacemos, construimos posibilidades. Lo que llamamos "ser" es un constante cambio; se es cambiando. No podemos, en adelante, considerar las cosas como entes aislados sino como sistemas que interactúan entre ellos y con el medio. Así nosotros formamos parte de todos los procesos, como seres en el mundo y como sujetos que pensamos el mundo, que tratamos de comprender y conocer ese mundo desde dentro de ese 
mundo. Nuestro gran enemigo es la mentalidad laplaciana (Roger Ciurana \& Regalado Lobo, 2016, pp. 49-59) o el idealismo que aísla pensamiento y experiencia.

La mayoría de los planes educativos se crean y se ponen en práctica con una conciencia metodológica positivista inconsciente: no se presta atención a los automatismos que la mayoría de las veces funcionan como distorsionantes epistemológicos inconscientes. En realidad, nuestro inconsciente epistemológico está poblado por un paradigma del orden que se manifiesta empíricamente en unas prácticas: determinismo, separación absoluta sujeto/objeto, negación del tiempo, metáfora de un conocimiento especular, observador laplaciano, negación del desorden o reducción a pura apariencia, etc. No se presta atención, decimos, a tantos mecanismos, rutinas, hábitos aprendidos y no reflexionados, que tal cual pasarán a ser base de "nuevos" planes de estudio. Todo ello sin darnos cuenta de que la innovación se debe reflejar antes en las cabezas de quienes van a elaborar los nuevos modelos educativos.

Por todo ello decimos que la pedagogía es un saber hermenéutico/práctico que, como cualquier saber social (o ciencia social) no es reducible a fórmulas matemáticas ni abstracciones idealistas. La pedagogía es un saber imperfecto, un trabajo imperfecto que nunca debería perder contacto con la realidad, una de cuyas características es la imprevisibilidad en muchos momentos. No se puede hacer una tarea educativa pertinente sin, al mismo tiempo, hacer una hermenéutica lo más ajustada posible del contexto.

Convendrá recordar esto en un tiempo en el que la exageración ingenieril y tecnocrática ${ }^{7}$ ahoga a las humanidades (se dice que porque no son útiles) y nos instala en la "seguridad" de los formalismos que se imponen como más reales que la realidad (o se presentan como los únicos reales, las únicas verdades enfrentadas a una realidad supuestamente falsa). Si es cierto que estamos entrando, o ya estamos en un mundo posdemocrático gobernado por la "posverdad", más que nunca estamos necesitados de pensar el modo como pensamos y vemos la realidad. Necesitados de pensar las construcciones y acercamientos que efectuamos en relación con el mundo. Porque se trata de 
distinguir del modo más claro posible lo que pensamos y lo que se nos da pensado; lo que vemos y lo que miramos más que lo que se nos presenta ya visto y mirado por grupos diversos de interés. Frente a tanta superficialidad necesitamos posicionarnos en una esfera de complejidad de pensamiento que reivindique un lenguaje lo menos manipulado posible.

Por medio de la educación y facilitado por los planes de estudio penetran concepciones del mundo, de la sociedad, del hombre. Se inyectan las ortodoxias, se seleccionan unos puntos, se silencian otros. Es decir, lo repetitivo y acumulativo es privilegiado en detrimento de lo flexible y creativo. Aún se requiere recordar, y ha pasado mucho tiempo ya, a M. Foucault (1974) cuando en su Lección Inaugural en el Collège de France (2 de diciembre de 1970) decía lo siguiente:

La educación, por más que sea, de derecho, el instrumento gracias al cual todo individuo en una sociedad como la nuestra puede acceder a no importa qué tipo de discurso, se sabe que sigue en su distribución, en lo que permite y en lo que impide, las líneas que le vienen marcadas por las distancias, las oposiciones y las luchas sociales. Todo sistema de educación es una forma política de mantener o de modificar la adecuación de los discursos, con los saberes y los poderes que implican. (p. 37).

Desde luego algo nos parece cierto, las adecuaciones se ejecutan por medio de los formatos curriculares que los enseñantes y los alumnos deben ejecutar. Recordamos lo que hemos escrito antes en relación con lo que se llama "excelencia” en la educación y su relación con el interés por el control.

A los autores de este texto no nos preocupa en principio el diseño curricular. Nos preocupa lo que está antes de la posibilidad de todo diseño: el para qué del diseño curricular. Se trata del problema básico del sentido y de la orientación. De la ideología que hay detrás, si queremos usar un término como el de "ideología", desprestigiado históricamente por muchos motivos, pero que tal como nosotros lo entendemos creemos que es interesante. Por “ideología” entendemos un sistema de ideas que por definición ni son buenas ni malas, otra cosa es el contacto entre ideas y realidad, para ello requerimos de la dimensión reflexiva, como decíamos antes. 
Nos preocupa lo que está antes de la posibilidad de todo diseño y ello nos convoca a todos a una reflexión sobre el sentido de la educación en la ciudad (polis). Conviene que no olvidemos que estamos hablando de un problema multidimensional: pedagógico, político, social, epistemológico. Estamos hablando en un problema de urbanización (Roger Ciurana \& Regalado Lobo, 2016, pp. 101 - 108) de la ciudad. Toda educación, de un modo u otro, desemboca en lo público, es una cuestión cívica ${ }^{8}$. Por ello mismo toda educación es ideológica. Porque toda educación propicia un orden, una visión de las cosas y de la sociedad en la que vivimos.

\section{Elucidación del sentido del currículo y los planes de estudio}

Un currículo es una puesta en diseño de ideas, una materialización de ideas que se manifiestan en los contenidos que presenta. Los contenidos de las materias curriculares, los modos como se codifiquen en las aulas las prácticas que se efectúen, van a generar una serie de aptitudes y valores en los alumnos. Las siguientes preguntas entonces se nos imponen: ¿Qué tipo de alumno deseamos que egrese de la institución educativa? ¿Qué perfil de alumno? ¿Qué perseguimos como educadores? ¿Somos meros replicantes de códigos y normas? ¿Aplicamos un currículo en abstracto? ¿Se trata de una cuestión meramente burocrática ejecutable por no importa qué docente ${ }^{9}$ ? Podríamos resumir todo en cuatro preguntas: ¿qué enseñar? ¿cómo enseñar? ¿para qué enseñar? ¿cuándo/dónde enseñar?

El qué son los contenidos, el cómo es la metodología, el para qué son los objetivos/habilidades y el cuándo/dónde hace referencia al contexto. No estamos, en ese sentido, muy lejos de las necesidades que, para el buen desempeño del ciudadano, habría que implementar: aprender a aprender, aprender a hacer, aprender a ser, aprender a vivir en sociedad. Todo ello requiere de otro tipo de estructura de pensamiento que vaya más allá de lo lineal, lo fragmentador, lo unidimensionalizante, lo que destruye y no articula. Porque necesitamos de modo vital poder convivir juntos en un mundo diverso y ello implica ir más allá de las lógicas de lo estático y de todo tipo de sustancialismos y esencias. El ser es 
creación producto de organización y auto-eco-re-organización, no tiene nada que ver con una sustancia ni identidad estática.

Nosotros pensamos que, si la educación es un laboratorio de materialización de ideas y de habilidades para una pertinente inserción del ser humano en su contexto, el asunto es sobre todo epistemológico (no olvidemos que la acción política es inseparable de un pensar, de una hermenéutica del contexto) y complementario con unas ideas faro hacia donde se dirige una determinada educación. Si estamos hablando de una materialización de ideas es importante que esas ideas se contextualicen, porque en abstracto no hay posibilidad de materialización. Por lo tanto, un currículo debe ser pensado como espacio de articulación y no a modo de enciclopedia (salvo que entendamos por "enciclopedia" saber en ciclo, en movimiento y articulación y no un saber que se apila sin relación). Se trata de articular los conocimientos en función de los problemas y las necesidades y no en función de concepciones rígidas y abstractas. Nuestros planes de estudio se quedan obsoletos porque no tienen en cuenta el tiempo. No se insertan en la historia y el movimiento. Es por ello por lo que cuando se habla de "innovar" sólo se aplica el término a la tecnología, pero no a la capacidad del sujeto para generar estrategias de pensamiento que construyan orden y sentido en el devenir de una realidad que produce muchas emergencias e imprevistos. En cambio, nuestros contenidos educativos se aplican en el aula como si el mundo fuese estático y no ocurriese nada. Evidentemente, en un mundo estático solo se necesitan programas y repeticiones, no hace falta ser creativos. Más aún, tenemos la receta predeterminada y el programa aplicable independientemente de la realidad a la que se aplica. Esa es la cultura del manual.

Pensemos un poco la fenomenología de la actualidad ya que estamos defendiendo la necesidad de que los planes de estudio se relacionen con el contexto para no seguir anclados en una educación con grados de abstracción tan grandes que la hacen impertinente. Además, difícilmente vamos a poder gestionar los problemas si antes no comprendemos su naturaleza. No podemos proponer un método separado de su objeto y circunstancia, pues vivimos en sociedades complejas. ¿Por qué las denominamos complejas?, porque constantemente emergen nuevas situaciones que se añaden a las existentes, se yuxtaponen, se entrelazan, 
chocan, en-redan, etc., creando un tejido dinámico en el tiempo. Se trata de una realidad compleja ${ }^{10}$, una realidad fenomenológicamente compleja, así nos aparece ("fenómeno" etimológicamente procede del griego fainomenon, lo que aparece, lo que se manifiesta). Una realidad que no podemos conocer de un modo exacto y total, no la podemos conocer de un modo totalmente objetivo y, por lo tanto, no podemos eliminar de ese conocimiento el componente hermenéutico, interpretativo, constructor del sujeto. Digamos en este momento algo que creemos importante, el problema de la teoría del conocimiento no es el de explicar el conocimiento del objeto sino el del conocimiento del conocimiento del objeto: se trata de profundizar en una epistemología del sujeto observador. Porque aquello a lo que llamamos "objeto" lo es porque un sujeto epistémico construye su "objetividad" a partir de las relaciones, articulaciones, etc., que ejecuta.

Vivimos en sociedades complejas que se caracterizan, abundando en lo que acabamos de decir, por su pluralidad, su multidimensionalidad, su irreductibilidad explicativa a un solo nivel: el problema de la multiculturalidad, el problema de la sanidad, el problema de la crisis económica, la educación cívica, los asuntos políticos y morales, son problemas que no se explican ni se resuelven desde un solo nivel de base. No podemos gestionar esos problemas desde formas de pensamiento monológicas y lineales. No podemos esencializar ninguno de los niveles ni perspectivas. Pero sí podemos comunicar y articular niveles para comprender mejor antes de actuar.

Nuestra realidad fenomenológica necesita de un nivel de complejidad epistemológica adecuado a esta realidad. Nos parece conveniente situarnos en ese nivel: no se trata tanto de una complejidad objetiva cuanto de una complejidad subjetiva. El problema de fondo es el problema de la mirada. El cómo gestionamos mentalmente la complejidad fenoménica. Qué mirada epistemológica aplicamos. Se trata de aumentar complejidad mental (epistémica) para reducir (no eliminar en su totalidad, es imposible) complejidad fenoménica. Sólo así creemos que es posible avanzar. Cuando decimos "reducir complejidad fenoménica" hablamos de gestionar y comprender; de leer la realidad creando otro tipo de conexiones conceptuales. En 
ese sentido se pueden aportar habilidades y aptitudes por medio de la educación que nos permitan organizar las cosas de otro modo. Así podemos salir del marco cognitivo dominante, o al menos revisarlo y, si fuese necesario, desecharlo. Si prestamos atención a lo que nos dicen Bateson, Morin, Bauman, Foucault, Castoriadis, von Foerster, etcétera, la comprensión de lo complejo, lo líquido, es cuestión también de crear nuevos marcos mentales abiertos para que tengan cabida las nuevas emergencias. En ese sentido necesitamos dirigirnos hacia la creación de marcos cognitivos abiertos que integren la dialógica compleja (la complementariedad de procesos o de niveles que, por separado, son excluyentes, pero pensándolos en conjunto nos sitúan en otro nivel de comprensión abierto, no en una síntesis que acaba con los procesos de confrontación) y el tiempo. Pensar de otro modo, crear nuevos vínculos, nuevas articulaciones que nos ayuden a generar nuevas imágenes para organizar de otro modo la experiencia.

Hoy podemos por experiencia decir que visiones deterministas y reduccionistas que derivan en acciones deterministas, reduccionistas, ciegas a lo empírico, producen resultados, la mayoría de las veces, desastrosos. El repetitivo e impuesto discurso de la globalización neoliberal, por ejemplo, en su visión economicista unidimensionalizante ha roto el mundo, ha contribuido a destruir relaciones sociales, culturales, etcétera. Los discursos culturalistas contribuyen a la imposibilidad del diálogo y la relación. Los nacionalismos esencialistas contribuyen a rupturas de relaciones, a separaciones entre aquellos que con una formación cultural en lo multidimensional podrían vivir en una unidad en la diversidad, en una unitas multiplex (Morin, 1977, p. 145). Hoy podemos decir por experiencia que actitudes que se basan en discursos monológicos y no se fundamentan en una cultura de la articulación y la organización son incapaces de gestionar las presentes complejidades en las que vivimos.

La mala educación basada en lo onto-lógico trata de imponer programas a la realidad para embridarla, someterla, sojuzgarla, congelarla. Una labor inteligente podría ser, en cambio, aquella que trata de producir "ajustes" y dialógicas con la realidad fenoménica. Aquella que trata de buscar crear sentidos nuevos en la gestión epistemológica de lo complementario y antagonista en un mismo espacio y tiempo; en la gestión dialógica del orden y el desorden. La realidad no nos aparece siempre con la misma forma invariante. La 
realidad es procesual. Necesitamos habilitarnos para gestionar procesos, no para gestionar lo estático y lo normal, lo repetitivo. Esto último se gestiona solo, con programas. Gestionar lo emergente y lo incierto no implica reducirlo a un sólo nivel o anularlo. Gestionar lo emergente y la novedad implica dia-logarlo, meternos en perspectiva. Un epistemólogo, en el sentido en que nosotros lo comprendemos, es un dialogador de perspectivas no un creador de monólogos.

Lo "nuevo" no es solo la novedad que emerge fenomenológicamente. Para nosotros lo "nuevo" es pensar de otro modo, articular, hacer otro tipo de luz. Innovar es, por lo tanto, innovar en el pensar para gestionar del modo más adecuado posible lo real. Es por lo que antes decíamos que se trata de la necesidad de una complejidad subjetiva. Decir "subjetivo" no equivale al nada es objetivo, al todo vale. Lo que queremos decir es más profundo: sin la intervención cognitiva del sujeto la realidad es bien poca cosa, no nos dice nada. Se trata de que se complejice mentalmente el sujeto para dialogar/comprender/gestionar la complejidad fenoménica. En ese sentido quizás podríamos hablar de una racionalidad no clásica, es decir, una racionalidad que se sitúe por encima de las dicotomías, las yuxtaposiciones, las reificaciones. Una racionalidad que vaya más allá del paradigma del orden. Una racionalidad no clásica, entonces, que incluya el desorden y la temporalidad, la irreversibilidad, el evento o acontecimiento. Una racionalidad no clásica que se sitúe por encima de las abstracciones y los absolutismos. Se trata, para nosotros, de una racionalidad auto-organizacional en relación con el contexto. Una racionalidad auto-eco-re-organizacional (Morin, 1980). Racionalidad a la que H. A. Simon (1983) denominaría racionalidad "plena" por contraste con una racionalidad limitada ${ }^{11}$. Se trata de poner en práctica una racionalidad compleja, activa frente a una racionalidad pasiva, disciplinaria, reduccionista, sin tiempo. Una racionalidad entendida como un procedimiento a la búsqueda de una verdad conversada que surge de una reflexión sobre la acción en el contexto frente a una verdad categórica, abstracta e inamovible. 
Es importante que reflexionemos sobre lo siguiente: estamos aún muy inmersos en la ilusión de que la razón y la acción racional se determinan de modo lineal y unidireccional. Por el contrario, nosotros pensamos que una acción racional formal y descontextualizada es inoperante para la gestión de los problemas reales. Por lo tanto, una educación en la que los planes de estudio tengan un alto grado de formalización no nos ayuda mucho para tratar las perturbaciones del medio. Coincidimos con Dewey (2004) cuando escribe lo siguiente:

La actitud filosófica es general en el sentido de que es opuesta a tomar nada aisladamente, trata de colocar un acto en su contexto, que es lo que constituye su significado... El pensar, por otra parte, es prospectivo en su referencia. Se ocasiona por una falta de asentamiento y aspira a vencer una perturbación. La filosofía consiste en pensar lo que lo conocido exige de nosotros, lo que la actitud de respuesta exige. Es una idea de lo que es posible, no el registro de un hecho cumplido. De ahí que sea hipotética como todo pensar. Presenta una asignación de algo que se ha de hacer, de algo que se ha de intentar. Su valor no está en proporcionar soluciones (lo cual solo puede conseguirse en la acción) sino en definir dificultades y sugerir métodos para vencerlas. (p. 274).

El método ${ }^{12}$ es entonces el movimiento de aprendizaje/reaprendizaje reorganizante entre los planes de estudio y la experiencia. Planes de estudio que aprenden de la experiencia y por retroacción nos sirven para dar nuevos sentidos a la experiencia, nos habilitan para redirigir las siguientes experiencias. Es por ello que hemos insistido en que la educación y los contenidos educativos deben huir de lo rutinario, lo programado. La educación es una constante reconstrucción. El problema es que cuando lo formal, lo reglado, lo controlado, se impone sobre lo empírico la experiencia educativa de aprendizaje/reaprendizaje es imposible.

La pedagogía es construcción de posibilidades para la creación y recreación del pensamiento de los sujetos, es decir, creación y recreación de sujetos capaces de gestionar su mundo de la manera más pertinente posible. Sin embargo, existen pedagogías que solo insertan modos de pensar específicos. En ese sentido se puede pensar en pedagogías sujetadoras de los sujetos a un determinado orden sólido y estático y, obtendríamos con ello, unos sujetos educados bajo un diseño curricular en el que a cada contenido adscrito al currículo le corresponden conceptos y objetos de análisis compartimentados y abstractos. El resultado es el siguiente: cuando el sujeto así educado afronta la realidad fenoménica busca objetos aislados, sin relación, a los que adherir sus conceptos. Una relación especular 
concepto/objeto, en síntesis. El costo de todo ello: eliminar las singularidades que los fenómenos adquieren en un espacio/tiempo específico; eliminar las interrelaciones entre contextos y dentro de los contextos. Todo ello a nivel ético se resuelve en la falta de pensamiento en torno a las múltiples consecuencias de la acción sobre el objeto en un contexto específico.

Desde nuestra perspectiva epistemológica planteamos esta pregunta: ¿Qué pasaría si además de pensar en las premisas que sustentan el diseño curricular el alumno/sujeto aprendiera a construir conocimiento a partir de los conocimientos contenidos en el currículo y en relación con los contextos? ¿Qué pasaría si el alumno aprendiera a repensar los conceptos y sus relaciones desde varias ópticas tratando de no olvidar el origen de esos conceptos y las preguntas que trataban de resolver en un espacio/tiempo específico? Ello nos llevaría a pensar en términos de currículos abiertos en los que se producen mezclas con otras disciplinas que pueden posibilitar nuevos conocimientos no por adición sino por redisposición y re-organización.

Para ello es necesario aprender estrategias de pensamiento que nos permitan construir de otro modo para vencer las perturbaciones que emergen en un contexto móvil en el que lo aleatorio y lo incierto son ineliminables en su totalidad. Lo que debe, a nuestro juicio, ir desapareciendo es una pedagogía en la que su principal intención sea la jerarquización de objetos sólidos, su visión aislada de las cosas, su ordenamiento. Una pedagogía basada en la lógica identitaria que organiza el mundo de un modo militar, clásico (no olvidemos que la palabra "clásico" hace referencia en su origen a la disposición de los soldados. En el ejército la palabra "classis" hace referencia a la flota, la escuadra de guerra).

Se trata de dirigirnos hacia la gestación de currículos que más que transferir orden estático, descontextualizado y sin historia, posibiliten la construcción de conocimientos abiertos y reorganizantes. 
Cada contenido curricular, cada concepto, cada objeto que será aprendido no es un ente aislado. Por el contrario, podemos concebir un eje de interrelaciones innumerables. En ese sentido la pedagogía que emerge de una concepción fluida de los fenómenos a través del espacio y del tiempo debe diseñar estrategias para el aprendizaje de lo multi-relacional, lo multi-causal, lo multi-dimensional que hay en los contenidos propuestos en el currículo. Contenidos siempre abiertos y ligados a un contexto local y global. Al mismo tiempo esos contenidos deben estar articulados a una reflexión ética sobre la relación de nuestras acciones y el mundo, pensada esta reflexión de un modo sistémico: los “objetos" de los que se ocupa la pedagogía son sociales, políticos, culturales, biológicos... En su interacción sostienen la vida colectiva e individual de los seres humanos. Por el contrario, un pensamiento unilineal y unicausal al invisibilizar estas relaciones impide pensar la complejidad de la acción y nos lleva a valoraciones simplistas de fenómenos interrelacionados. No se puede comprender una acción si no la comprendemos como acción ecologizada: acción en la que intervienen y se entrecruzan muchas líneas de acción.

\section{Conclusión}

Desde nuestra perspectiva, repetimos, epistemológica, sugerimos que el currículo conciba al sujeto que aprende no como un sujeto repetidor de un saber sino como un sujeto capaz de reorganizar y, por lo tanto, recrear y valorar los saberes aprendidos. Capaz de transmutar estos saberes en acciones pertinentes sobre el mundo. En ese sentido es como podremos comprender la idea de que toda teoría que no se pueda concretar en una práctica es una teoría innecesaria, inútil.

En realidad, la tarea de la educación es que no nos perdamos en las insignificancias. Que podamos vivir en la creatividad, lejos de la concepción del sujeto como un depósito de datos y habilidades, repetidor de una realidad; lejos de lo estático, lejos del fiasco que supone el especialismo desconectado. Porque un especialista sólo tiene posibilidades en contextos muy triviales. No se trata, por el contrario, de ser unos generalistas que desconocen las partes, se trata de ser complejos entendiendo por ello lo siguiente: una epistemología compleja es producto de una dialógica de perspectivas. Se trata de habilitar, de crear condiciones 
pedagógicas para la emergencia de mentes transdisciplinares capaces de poder establecer un dialogo creativo de perspectivas. El sujeto/epistemólogo entonces pasa a ser un creador de unidad en la diversidad, un dialogador de perspectivas. Porque el conocimiento emerge a partir de la articulación de lo multidimensional. Todo ello es otro modo de decir que lo importante es conversar, porque la conversación es el único modo que tenemos los humanos para poder transitar por los espacios de la incertidumbre, es decir, transitar por la vida siendo creadores.

\section{Notas}

${ }^{1}$ En otra parte hemos definido lo que para nosotros es la "innovación” de este modo: "por innovación entendemos la capacidad de ofrecer otros medios de configurar y de abordar las cuestiones que definen nuestras actitudes y nuestros comportamientos. La innovación es la capacidad de liberar otras posibilidades de comprensión y acción más ajustadas, más pertinentes en relación con el o los contextos. Innovación como habilidad de incorporar otras perspectivas en un mundo en el que emerge constantemente la incertidumbre y hace necesario un sujeto - estratega en la capacidad de auto-eco-re-organización permanente”. (Roger Ciurana \& Regalado Lobo, 2016, pp. 18-19)

${ }^{2}$ Dice así Bauman: "los llamamientos sistemáticos a que el «desarrollo de recursos humanos» basado en la «detección de las aptitudes requeridas por el mercado de trabajo» ejerza un papel director en la materia han sido innumerables en el pasado y en el momento presente. Sin embargo (y con una regularidad igualmente monótona) los gestores de «recursos humanos» se han visto una y otra vez incapaces de prever lo que el «mercado laboral» iba a «necesitar» cuando la «fuerza laboral» actualmente en formación finalizara su periodo de instrucción y estuviera presumiblemente preparada para trabajar. Nunca es fácil predecir los giros futuros de la demanda del mercado, por muy astuto que sea el pronosticador y por muy metodológicamente depurados que sean sus pronósticos". (Bauman, 2006, p. 163)

${ }^{3}$ Dice así Dewey: "la rutina, la acción que es automática, puede aumentar la habilidad para hacer una cosa particular. En este sentido, puede decirse que tiene un efecto educativo. Pero no lleva a nuevas percepciones de las consecuencias y conexiones; limita más que amplía el horizonte significativo. Y como el ambiente cambia y nuestro modo de actuar ha de modificarse para mantener con éxito una 
conexión equilibrada con las cosas, un modo uniforme aislado de actuar llega a ser desastroso en algún momento crítico. La alabada "destreza” se convierte en una gran ineptitud” (Dewey, 2004, p. 75). Remitimos también a nuestro texto Pensamiento complejo y Educación. Aclaraciones y confrontaciones (2016), en donde en las páginas 17 a 19 se reflexiona sobre el problema de lo programado y rutinario en relación con la sociología de las organizaciones. El peligro de lo rutinario y programado es que todo se convierta en un pensamiento burocratizado y controlador que crea que algo funciona cuando todo desorden o imprevisto es eliminado u ocultado para salvar la idea o la imagen de la realidad que tiene la institución. Un pensamiento burocratizado que trata de gestionar la realidad prescindiendo de la realidad. Efectivamente, una educación disociada de la fenomenología socio-cultural puede acabar convirtiéndonos en unos ineptos, inhábiles, incapaces de cualquier gestión pertinente.

${ }^{4}$ José Antonio Marina (2004) en su libro La inteligencia fracasada reflexionando sobre lo que denomina "los fracasos de la voluntad" entendida esta última como "la motivación inteligentemente dirigida", nos habla de la rutina y dice lo siguiente: "los hábitos son mecanismos aprendidos que automatizan ciertas conductas de tal modo que nos permiten realizarlas con mayor facilidad, perfección, y sin necesidad de que prestemos atención. Son indispensables para toda acción eficiente [...] Sin embargo, los hábitos pueden liberarnos. Pero también pueden esclavizarnos. Por eso deben estar sometidos a una vigilancia superior. Intentar resolver un problema de manera rutinaria sólo sirve cuando los problemas son muy elementales. El mecanismo puede dispararse automáticamente e intentar someter a su acción cualquier conflicto, en lugar de acomodarse él al conflicto. Los estudios sobre el fracaso de los directivos empresariales o de los políticos atribuyen muchos de ellos a la creencia de que lo que funcionó en un caso debe funcionar siempre” (pp. 98,113).

${ }^{5}$ Desde un punto de vista epistemológico hablamos de un principio dialógico. Se trata de pensar en un mismo espacio/tiempo una relación de lógicas que se complementan y que en un momento dado pueden excluirse. Se trata del entrelazamiento complejo de instancias que en su articulación complementaria/antagonista pueden hacer emerger nuevas formas incomprensibles desde una lógica de la fragmentación y la exclusión. Se trata de articular perspectivas para crear órdenes de comprensión más amplios.

${ }^{6}$ Creemos necesario acudir a las palabras que escribe E. Morin (1980) cuando distingue lo que es método como estrategia de pensamiento complejo de lo que es programa: "la oposición programa/estrategia salta a la vista. El programa constituye una organización predeterminada de la 
acción. La estrategia encuentra recursos y rodeos, realiza inversiones, desvíos. El programa efectúa la repetición de lo mismo en lo mismo, es decir, necesita condiciones estables para su ejecución. La estrategia es abierta, evolutiva, afronta lo imprevisto, lo nuevo. El programa no improvisa ni innova. La estrategia improvisa e innova. El programa solo puede experimentar una dosis débil y superficial de alea y de obstáculos en su desarrollo. La estrategia se despliega en aleatorias, utiliza el alea, el obstáculo, la diversidad, para alcanzar sus fines. El programa solo puede tolerar una dosis débil y superficial de errores en su funcionamiento. La estrategia saca provecho de sus errores [...] El programa necesita del control y de la vigilancia computante. La estrategia no solo necesita control y vigilancia, sino, en todo momento, competencia, iniciativa, decisión” (p. 225). También Morin, E. (1986, pp. 61-63)

${ }^{7}$ No podemos evitar recordar la descripción que Charles Dickens (2007) hace de su personaje Thomas Gradgrind, prototipo del utilitarista radical: “Thomas Gradgrind, sí, señor. Un hombre de realidades. Un hombre de hechos y números. Un hombre que arranca del principio de que dos y dos son cuatro y nada más que cuatro y al que no se le puede hablar del que consienta que alguna vez sean algo más. Thomas Gradgrind, sí, señor... Un señor con la regla, la balanza y la tabla de multiplicar siempre en el bolsillo, dispuesto a pesar y a medir en todo momento cualquier partícula de la naturaleza humana para decirnos con exactitud a cuanto equivale. Un hombre reducido a números, un caso de pura aritmética”. (pp. 86-87). ¿Tan alejados estamos hoy de esas palabras que escribe Dickens en 1854 ? ¿O quizás podamos decir aún, por desgracia, que la mentalidad utilitarista y mercantilista que denunciaba Dickens en la enseñanza de su época sigue hoy permeando muchas mentalidades en nuestras instituciones educativas? De hecho, el planteamiento pedagógico de la reforma educativa que está planteando la Secretaria de Educación Pública [SEP] (2016) del Gobierno Mexicano trata de introducir cambios educativos para atajar este problema que arrastra la educación: la falta de mentalidad humanista y la exagerada mentalidad positivista/utilitarista. Se trata de ir más allá del modelo educativo homogeneizante, jerárquico, negador de la diversidad y de la imaginación, negador de la creatividad. Se puede consultar sin problemas por internet el Modelo Educativo 2016 del que acabamos de hablar en: https://www.gob.mx/cms/uploads/attachment/file/118382/El_Modelo_Educativo_2016.pdf.

${ }^{8}$ De hecho, ya Platón, años antes de que Aristóteles reprodujese prácticamente la misma idea en su Política, escribe en el diálogo Las Leyes que su argumentación es "la de los que piensan en la 
educación para la virtud desde la infancia, que hace al niño deseoso y apasionado de convertirse en ciudadano, con saber suficiente para gobernar y ser gobernado en justicia. Definiendo nuestro argumento esta crianza, solo a ella, según creo, consentiría en llamar educación” (Las Leyes, 644a). Un hombre rectamente educado es un hombre bueno, nos dice Platón, un ciudadano. Y la educación es el primero de los más hermosos bienes.

${ }^{9}$ Un currículo no se ejecuta por sí solo. Creemos que el profesorado tiene la obligación de repensarse y de repensar el modo de comunicar los contenidos que imparte articulados con el contexto. No se trata de que llenemos de pensamientos la cabeza del alumno, se trata de enseñarles a pensar, enseñarles a ser inteligentes y saber relacionar pensamiento y experiencia. El alumno se convierte en un mero reproductor porque el profesor es un reproductor que pide al alumno que reproduzca y repita información. Pero una información desvinculada del entorno es una abstracción insensible, un fiasco. En la relación pedagógica se reproduce el paradigma del orden y la ideología de la fragmentación. Un pensamiento que trocea la realidad se reproduce disciplinariamente en el aula, cada materia que enseña el profesor es un trozo de realidad. El cambio metodológico hoy exige que pensemos de modo articulante, que establezcamos conexiones, que no sigamos instalados en el error de pensar que conocemos mejor y explicamos mejor fragmentándolo todo.

${ }^{10}$ Atención: no estamos planteándonos el tema a nivel ontológico, es decir, no se trata de resolver el enigma metafísico de si el mundo en su fundamento es simple o complejo.

${ }^{11}$ También puede consultarse Simon, H. A. (1989) el prefacio.

${ }^{12}$ En relación a nuestra idea de método, que no cabe confundir con metodología remitimos al Apéndice 2 del libro Pensamiento complejo y educación, aclaraciones y confrontaciones (Roger Ciurana \& Regalado Lobo, 2016, pp. 41-44), así como a la obra general de E. Morin de quien somos deudores en muchos aspectos. 
Referencias

Bachelar, G. (1981). El nuevo espíritu científico. México: Nueva Alianza.

Bauman, Z. (2006). Vida Líquida. Barcelona: Paidós.

Dewey, J. (2004). Democracia y Educación. Madrid: Ediciones Morata S.L.

Dickens, C. (2007). Tiempos difíciles. Madrid: Cátedra Letras Universales.

Foucault, M. (1974). El orden del discurso. Barcelona: Tusquets.

Marina, J. A. (2004). La inteligencia fracasada. Barcelona: Anagrama.

Morin, E. (2014). Enseigner à vivre. Manifeste pour changer l'education. Arles. Arles: ACTES SUD / PLAYBAC.

Morin, E. (1977). La Méthode 1: La nature de la nature. Paris : Seuil.

Morin, E. (1980), La Méthode 2, La vie de la vie. Paris : Seuil.

Morin, E. (1986) La Méthode 3, La connaisance de la connaissance. Paris: Seuil.

Platón. (2014). Las Leyes. Madrid: Alianza Editorial.

Roger Ciurana, E., \& Regalado Lobo, C. (2016). Pensamiento complejo y educación. Aclaraciones y confrontaciones. México: FronterAbierta.

Secretaría de Educación Pública (2016). Modelo Educativo 2016. Recuperado el 13 de noviembre de 2017, https://www.gob.mx/cms/uploads/attachment/file/118382/El_Modelo_Educativo_2 016.pdf

Simon, H. A. (1983). Reason in Human affairs. California: Stanford University Press

Simon, H. A. (1989). Naturaleza y límites de la razón humana. México: Fondo de Cultura Económica. 